\title{
Surface modifications of AISI 420 stainless steel by low energy Yttrium ions
}

\author{
Vincenzo Nassisi ${ }^{1}$, Domenico Delle Side, Vito Turco, Luigi Martina \\ Dipartimento di Matematica e Fisica "E. De Giorgi”, Università del Salento - Lecce, Via Provinciale Lecce-Monteroni, 73100 \\ Lecce - Italy, Tel. +39 0832 297495, Fax.+39 0832 297482, E-Mail: vincenzo.nassisi@le.infn.it
}

\begin{abstract}
In this work, we study surface modifications of AISI 420 stainless steel specimens in order to improve their surface properties. Oxidation resistance and surface micro-hardness were analyzed. Using an ion beam delivered by a Laser Ion Source (LIS) coupled to an electrostatic accelerator, we performed implantation of low energy yttrium ions on the samples. The ions experienced an acceleration passing through a gap whose ends had a potential difference of $60 \mathrm{kV}$. The gap was placed immediately before the samples surface. The LIS produced high ions fluxes per laser pulse, up to $3 \times 10^{11}$ ions $/ \mathrm{cm}^{2}$, resulting in a total implanted flux of $7 \times 10^{15}$ ions $/ \mathrm{cm}^{2}$. The samples were characterized before and after ion implantation using two analytical techniques. They were also thermally treated to investigate the oxide scale. The crystal phases were identified by an X-ray diffractometer, while the micro-hardness was assayed using the scratch test and a profilometer. The first analysis was applied to blank, implanted and thermally treated sample surface, while the latter was applied only to blank and implanted sample surfaces. We found a slight increase in the hardness values and an increase to oxygen resistance. The implantation technique we used has the advantages, with respect to conventional methods, to modify the samples at low temperature avoiding stray diffusion of ions inside the substrate bulk.
\end{abstract}

\section{Introduction}

Ion-implantation is an established method of surface modification of materials leading to an improvement of their mechanical proprieties e.g. micro-hardness, wear resistance and corrosion resistance [1, 2]. Alloys undergoing high temperatures are frequently used in power plants, gasification systems, petrochemical industry, combustion processes and in aerospace applications [3]. Depending on the application, materials are exposed to corrosive atmospheres and/or thermal cycling.

High temperature resistant alloys have to combine with two main requirements: low scale growing rate and adequate scale adherence on the alloy, especially if thermal cycles are considered. According to the literature, the control of the oxidation is particularly due to formation of $\mathrm{Cr}_{2} \mathrm{O}_{3}$ and $\mathrm{Al}_{2} \mathrm{O}_{3}$ [4-5].

The beneficial effects of active element additions on the oxidation resistance of heat resistant alloys are well known. Small amounts (usually below 1\%) of reactive elements (Sc, Ti, Y, Zr, Ce, La, ect.) clearly improve the oxidation behaviour of chromia- and alumina-forming alloys [6-7]. Several explanations are given about this effect, usually called reactive element effect (REE), namely a modification of the diffusion mechanisms, a reduction of vacancies condensation at the internal

\footnotetext{
Corresponding author: vincenzo.nassisi@unisalento.it
}

interface, a formation of reactive element oxide inclusions trapping alloy impurities [8-10].

In this work, thermal treatment was carried out in order to study the influence of implanted yttrium on the oxide scale adherence on 410 steel specimens oxidized at $1123 \mathrm{~K}$. We have chosen the yttrium, in particular, for its availability and its low cost.

$\mathrm{X}$-ray diffraction analyses indicate that the oxides formed near $1000 \mathrm{~K}$ are different on blank compared to implanted samples. Measurements of XRD allow analyzing the oxide scale composition after cooling to room temperature. Consequently, we also focus on the possible correlation between the presence of implanted yttrium and the oxide structure formed at high temperature.

\section{Experimental apparatus}

The experimental apparatus utilized for performing implantation of samples is an accelerator composed by a KrF excimer laser (Lambda Physics, Compex), operating at $248 \mathrm{~nm}$ wavelength (5 eV photon energy, total energy fixed at $11 \mathrm{~mJ} /$ pulse), $23 \mathrm{~ns}$ pulse duration to induce plasmas by PLA and a vacuum chamber made of stainless steel [11]. Inside the vacuum chamber, an expansion chamber is placed tightly closed around the target support. It was $18 \mathrm{~cm}$ in length and $8 \mathrm{~cm}$ in diameter. The target and the expansion chamber are connected to a power supply of positive polarity. The 
expansion chamber end has a $1.5 \mathrm{~cm}$ hole to allow ion extraction. A ground electrode was placed at $3 \mathrm{~cm}$ distance from the expansion chamber. After this electrode, a third electrode at $2 \mathrm{~cm}$ distance from ground electrode, connected to a power supply of negative polarity, acted as samples holder. The same electrode can also work as Faraday cup. In this configuration, the electrode connects to an oscilloscope using a high voltage capacitor $(2 \mathrm{nF})$, in order to separate the oscilloscope from high voltage, and a voltage attenuator, in order to decrease the electric signal. In Fig. 1 the stretch of the device is shown.

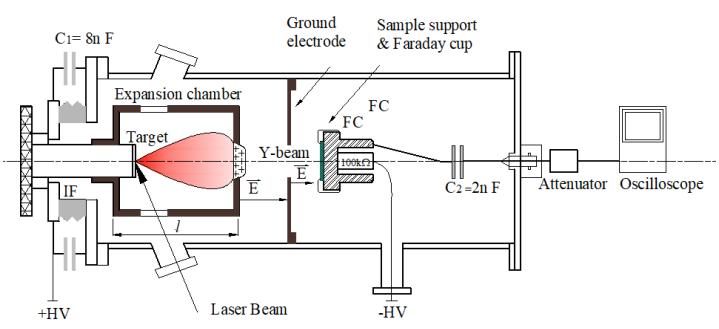

Fig. 1: Schematic drawing of the experimental apparatus.

During the experiments, we fixed the positive and the negative voltage respectively at $+40 \mathrm{kV}$ and $-20 \mathrm{kV}$. This corresponds to a maximum acceleration voltage of $60 \mathrm{kV}$. In this condition, the ion flux resulted of $3 \times 10^{11}$ ions $/ \mathrm{cm}^{2}$. For a deeper characterization of the ion beams, we remand the reader to the analysis in [12] and references therein.

The samples we used to perform oxidation experiments were foils of AISI 420 stainless steels. This is a widely used alloy, for example for surgical tools. Therefore, increasing the oxidation resistance of such material is very useful. The composition of the AISI420 is given in Table 1. All the samples utilized had a square shape (15 $\mathrm{mm} \times 15 \mathrm{~mm}$ ) and a thickness of $2 \mathrm{~mm}$.

Table 1: Chemical composition (wt $\%$ ) of the stainless steel: AISI 420

\begin{tabular}{|l|r|}
\hline \multicolumn{2}{|l|}{ Chemical composition (wt\%) } \\
\hline $\mathrm{Fe}$ & 86.7 \\
\hline $\mathrm{Cr}$ & 13.0 \\
\hline $\mathrm{C}$ & 0.3 \\
\hline
\end{tabular}

In the described experimental conditions, we obtain mainly yttrium ions with charge states +1 and +2 , with a small presence of +3 states. This translates to an ion range inside the samples ranging from 15 to $40 \mathrm{~nm}$.

The scratch tests were performed with a diamond tip on which a $0.60 \mathrm{~N}$ perpendicular force was applied. This produced a scratch on the samples surface and we measured the scratch width and depth. For the roughness measurements, we used the stylus surface profiler (Tencor Instruments ALPHA-STEP 200), which determined the arithmetic surface roughness using the graphical-centerline method. The stylus surface profiler was set with an applied force of $0.12 \mathrm{mN}$, a scan length of $2000 \mu \mathrm{m}$ and a scan speed of $100 \mu \mathrm{ms}-1$.
Oxidation behavior was investigated by utilized a classic muffle furnace with controller (GEFRAN 400, GEFRAM S.p.A.) at atmospheric pressure in air. Even treatment was performed putting the sample in a porcelain crucible at a temperature of $1123 \mathrm{~K}$ for 24 hours. After the thermal treatment and cooling to room temperature the samples were analyzed by an XRD (Rigaku D/MAX Ultima+).

\section{Experimental results}

Before implantation, we operated an accurate cleaning of samples in ethanol. The samples were then implanted on one face, which corresponds to approximately $70 \%$ of the total surface area due to the mask utilized to hold the samples on the support. The final implanted flux was reached applying 22000 laser pulses, resulting in $7 \times 10^{15}$ ions $/ \mathrm{cm}^{2}$.

After implantation, we measured the micro-hardness characteristics, using the "scratch test" method. Fig. 2 shows the scratch test results of our samples. The scratch test results shows that implanted samples exhibit scratches with lower depth $(\sim 0.2 \mu \mathrm{m})$ with respect to blanks $(\sim 0.5 \mu \mathrm{m})$. Therefore, the smaller scratch points out a lower tip depth in the sample surface, i.e. a sensible, although little, increment of the surface microhardness occurred in the implanted sample.

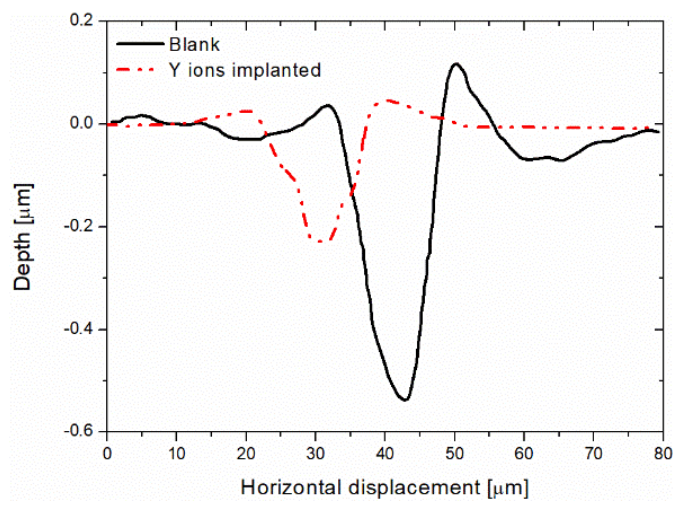

Fig 2: Profile of the scratch on the blank (black solid line) and on the Y-implanted sample (red un-solid line).

The XRD spectra of the blank and Y-implanted sample did not show significant differences and both exhibited mainly iron peaks, Fig. 3. Instead, the thermally processed samples (blank and Y-implanted) exhibited, apart the iron peaks, also many peaks related to the oxide families. In fact, by close examination, it can be concluded that $\mathrm{Cr}_{2} \mathrm{O}_{3}$ (Joint Commitee Powder Diffraction Standard file: JCPDS no. 38-1479) is the most probable oxide in the scale. Before furnace treatment, we observed also presence of $\mathrm{Fe}_{2} \mathrm{O}_{3}$ nuclei. Other corrosion products, mainly $\mathrm{Cr}_{2} \mathrm{O}_{3}$ and $\mathrm{Mn}_{1.5} \mathrm{Cr}_{1.5} \mathrm{O}_{4}$, then covered them. The signal intensities of $\mathrm{Cr}_{2} \mathrm{O}_{3}$ were different for the two cases analyzed. Fig 4a shows the spectrum of the XRD analysis for the blank sample, while Fig. $4 \mathrm{~b}$ shows the spectrum of the XRD analysis for the $\mathrm{Y}$-implanted sample. The intensity (cps) of the $\mathrm{Cr}_{2} \mathrm{O}_{3}$ signal for the blank sample was higher than 
the implanted one. In fact the signal of $\mathrm{Cr}_{2} \mathrm{O}_{3}$ at $2 \theta=24.38^{\circ}$ is 209 for the blank sample, while it is 43 for the Y-implanted sample.

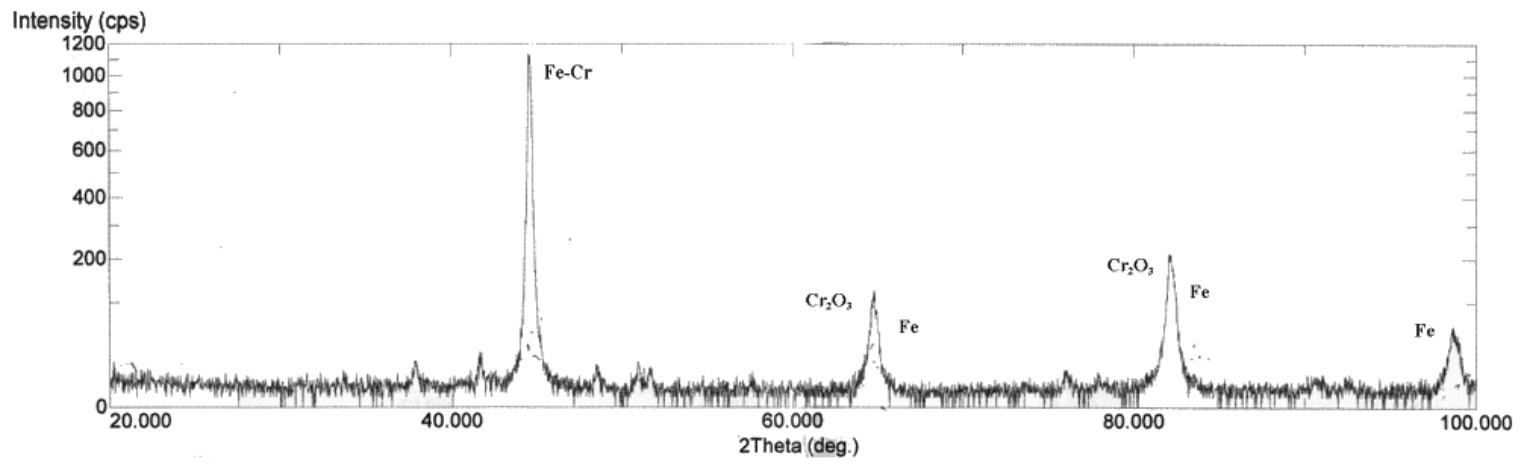

Fig. 3: XRD spectrum of the blank and Y-implanted samples before thermal treatment
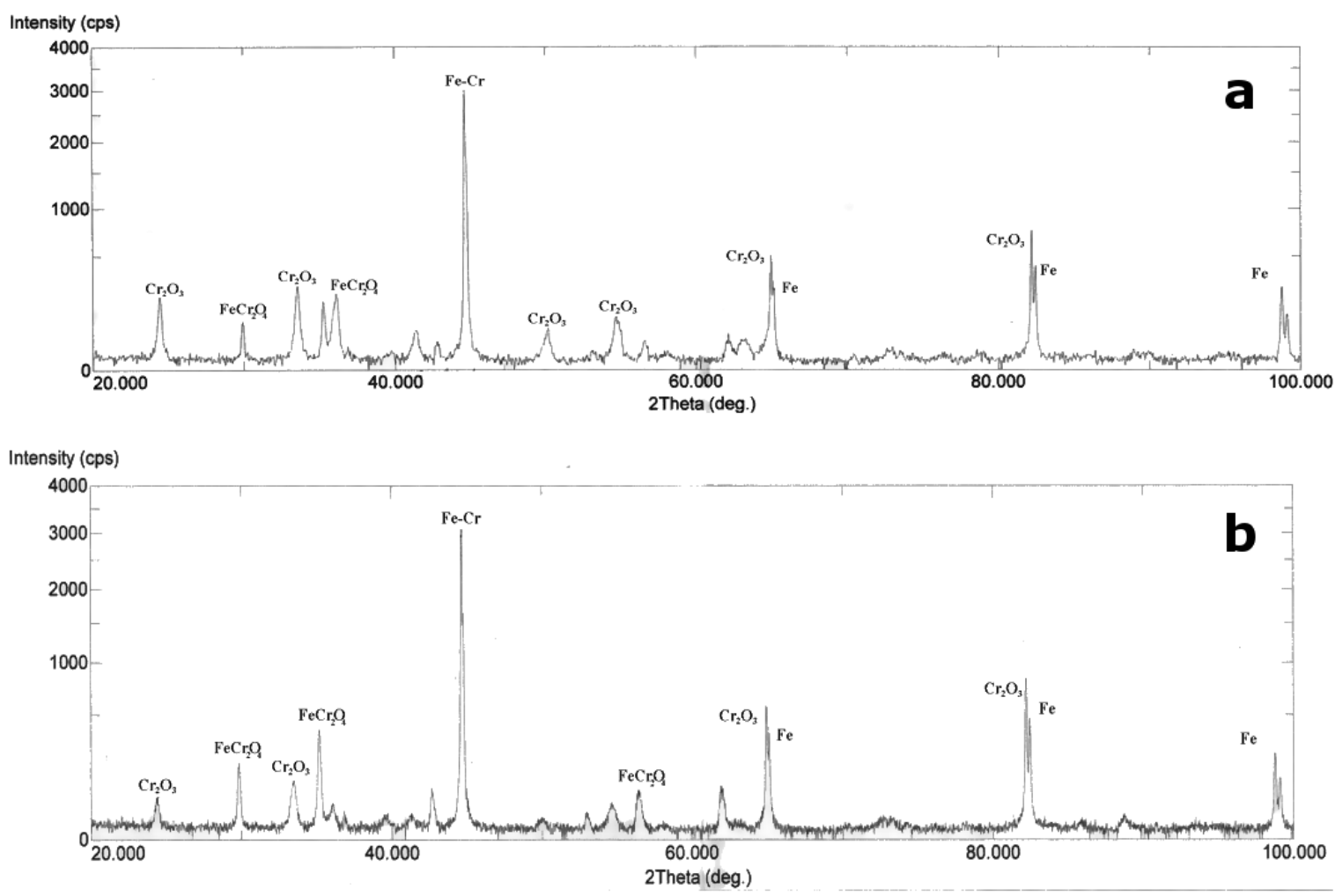

Fig. 4: XRD spectrum after thermal treatment. a) blank sample; b) Y-implanted sample.

We can observe that the implantation of $Y$ ions modify the characteristic of the stainless steel samples. As proposed by other authors [13-16], we have shown that yttrium implantation also induce some surface alloy structural modification promoting partial phase transformation of austenite into bcc structure ( $\alpha$-ferritic) near the surface. Even if any yttrium containing oxides have not been observed by XRD (Fig. 4b), results show that yttrium reduces the oxidation rate.

\section{Conclusions}

We found that implantation of low energy yttrium ions is able to increase hardness of AISI 420 stainless steel samples. XRD measurements further pointed out that an enhancement of the oxidation resistance is also present. In the experiment we used just one dose. We can suppose that at higher doses hardness and oxidation resistance could improve. This treatment is simple and ensures effective results on the AISI 420 surfaces. 
Consequently, it could represent a valuable tool for improvements of AISI 420 surface properties.

\section{References}

1. G. Dearneley, Nucl. Instr. and Meth. 182-183 (1981) 899.

2. M.J. Bennett, A. T. Tuson, Mat. Sci. Eng. A 116 (1989) 79.

3. F.J. Perez, M.J. Cristobal ,M.P. Hierro ,F. Pedraza Surf. \& Coat. Tecnol. 120 (1999) 442.

4. C. Wagner, Atomic Movements, American Society of Metallurgy, Cleveland, 1951, p. 153.

5. M.J. Bennet, D.P. Moon, in: E. Lang (Ed.), The Role of Active Elements in the Oxidation Behaviour of High Temperature Metals and Alloys, Elsevier, Amsterdam, 1989, p. 111.

6. M. Landkof, A.V. Levy, D.H. Boone, R. Gray, E. Yaniv, Corros.-NACE 41 (1985) 344.

7. D.P. Whittle, J. Stringer, Philos. Trans. R. Soc., Lond. Ser. A 295 (1980) 309.

8. F.I. Wei, F.H. Stott, Corros. Sci. 29 (1989) 839.

9. P.Y. Hou, J. Stringer, Oxid. Met. 34 (1990) 299.

10. E.A. Polman, T. Fransen, P.J. Gellings, Oxid. Met. 33 (1990) 135-155.

11. Velardi, L., Siciliano, M. V., Delle Side, D., \& Nassisi, V., Rev Sci Instrum 83(2) (2012) 02B717.

12. Nassisi V., Velardi L. and Delle Side D., Appl Surf Sci, 273 (2013) pp. 114-118.

13. R. Guillamet, J. Lopitaux, B. Hannoyer, M. Lenglet, J. Phys. IV C9 (3) (1993) 349.

14. M. Guemmaz, A. Mosser, J.J. Grob, R. Stuck, Surf. Coat. Technol. 100-101 (1998) 353.

15. A. Gutierrez, M.F. Lopez, F.J. Perez-Trujillo, Surf. Interface Anal. 30 (2000) 130.

16. G. Xie, M. Song, K. Mitsuishi, K. Furuya, J. Nucl. Mater. 281 (2000) 80 